\title{
Clinical Efficacy of Various Diagnostic Tests for Small Bowel Tumors and Clinical Features of Tumors Missed by Capsule Endoscopy
}

\author{
Jung Wan Han, ${ }^{1}$ Sung Noh Hong, ${ }^{2}$ Hyun Joo Jang, ${ }^{1}$ Seong Ran Jeon, ${ }^{3}$ \\ Jae Myung Cha, ${ }^{4}$ Soo Jung Park, ${ }^{5}$ Jung Sik Byeon, ${ }^{6}$ Bong Min Ko, ${ }^{7}$ Eun Ran Kim, ${ }^{2}$ \\ Hwang Choi, ${ }^{8}$ and Dong Kyung Chang ${ }^{2}$ \\ ${ }^{1}$ Division of Gastroenterology, Department of Internal Medicine, Dongtan Sacred Heart Hospital, \\ Hallym University College of Medicine, 40 Sukwoo-dong, Hwaseong-si, Gyeonggi-do 445-170, Republic of Korea \\ ${ }^{2}$ Department of Gastroenterology, Samsung Medical Center, Sungkyunkwan University School of Medicine, Seoul, Republic of Korea \\ ${ }^{3}$ Department of Gastroenterology, Seoul Soon Chun Hyang University Hospital, Soon Chun Hyang University College of Medicine, \\ Seoul, Republic of Korea \\ ${ }^{4}$ Department of Gastroenterology, Kyung Hee University Hospital at Gangdong, Kyung Hee University College of Medicine, \\ Seoul, Republic of Korea \\ ${ }^{5}$ Department of Gastroenterology, Yonsei University College of Medicine, Seoul, Republic of Korea \\ ${ }^{6}$ Department of Gastroenterology, Asan Medical Center, University of Ulsan College of Medicine, Seoul, Republic of Korea \\ ${ }^{7}$ Department of Gastroenterology, Bucheon Soon Chun Hyang University Hospital, Soon Chun Hyang University College of Medicine, \\ Bucheon, Republic of Korea \\ ${ }^{8}$ Department of Gastroenterology, Incheon St. Mary's Hospital, Catholic University School of Medicine, Incheon, Republic of Korea
}

Correspondence should be addressed to Hyun Joo Jang; jhj1229@hallym.or.kr

Received 30 March 2015; Revised 20 May 2015; Accepted 25 May 2015

Academic Editor: Hsu-Heng Yen

Copyright (C) 2015 Jung Wan Han et al. This is an open access article distributed under the Creative Commons Attribution License, which permits unrestricted use, distribution, and reproduction in any medium, provided the original work is properly cited.

Background. We aimed to evaluate the efficacy of various diagnostic tools such as computerized tomography (CT), small bowel follow-through (SBFT), and capsule endoscopy (CE) in diagnosing small bowel tumors (SBTs). Additionally, we aimed to evaluate the clinical features of SBTs missed by CE. Methods. We retrospectively studied 79 patients with histologically proven SBT. Clinical data were analyzed with particular attention to the efficacy of CT, SBFT, and CE in detecting SBT preoperatively. We also analyzed the clinical features of SBTs missed by CE. Results. The most common symptoms of SBT were bleeding (43\%) and abdominal pain (13.9\%). Diagnostic yields were as follows: CT detected 55.8\% of proven SBTs; SBFT, 46.1\%; and CE, 83.3\%. The sensitivity for detecting SBTs was $40.4 \%$ for CT, $43.9 \%$ for SBFT, and 79.6\% for CE. Two patients with nondiagnostic but suspicious findings on CE and seven patients with negative findings on CE were eventually found to have SBT. These nine patients were eventually diagnosed with gastrointestinal stromal tumor (4), small polyps (3), inflammatory fibroid polyp (1), and adenocarcinoma (1). These tumors were located in the proximal jejunum (5), middle jejunum (1), distal jejunum (1), and proximal ileum (1). Conclusion. CE is more efficacious than CT or SBFT for detecting SBTs. However, significant tumors may go undetected with CE, particularly when located in the proximal jejunum.

\section{Introduction}

The small intestine represents $75 \%$ of the length and $90 \%$ of the absorptive surface area of the gastrointestinal system. However, small bowel tumors (SBTs) are rare, representing only 3-6\% of gastrointestinal (GI) tract tumors and only 1$3 \%$ of all malignant GI tumors [1]. The diagnosis of SBTs is difficult and is frequently delayed. Conventional diagnostic modalities are inaccurate and inconclusive [2] and frequently fail to detect early or locally advanced stages because of 
the inaccessibility of the small bowel. Indirect evaluation is possible with small bowel follow-through (SBFT) or computed tomography (CT), but disadvantages include patient discomfort, high radiation dose, and, most importantly, poor sensitivity in detecting SBTs $[3,4]$.

Capsule endoscopy (CE) allows painless endoscopic imaging of the entire small bowel. CE has rapidly gained acceptance as a standard method for small bowel evaluation even though it is contraindicated in patients with suspected or documented intestinal obstruction and does not allow therapeutic intervention $[5,6]$. In large cohort undergoing $\mathrm{CE}$, the prevalence of SBT ranges from 2 to $9 \%$, higher than previously reported [7-14]. CE may provide the earlier diagnosis and treatment of SBTs compared to comparative methods $[8,15]$. However, some studies revealed that CE can miss some significant tumor in the small bowel [16-18].

This study aimed to investigate the characteristics of SBTs confirmed by pathology, to compare diagnostic yields among various diagnostic methods, and to identify the characteristics of SBTs missed by CE.

\section{Materials and Methods}

The records of 79 patients with histology proven SBTs by surgery (43 patients) or double balloon enteroscopy (DBE, 65 patients) from March 2004 to December 2012 were retrospectively studied. Patients had been treated at 7 medical referral centers in Korea and had undergone surgery or DBE for SBT removal. We reviewed the clinical characteristics of SBTs; the diagnostic yields of SBFT, CT, and CE; and the characteristics of SBTs not detected by CE. Of these 79 patients, 39 (49.3\%) underwent SBFT, 68 (86.1\%) underwent CT, and 54 (68.3\%) underwent CE before SBTs were confirmed. Findings of these diagnostic studies were interpreted by experienced endoscopists and radiologists, and the results were classified into three categories: definite, suspicious, or negative. When SBFT, CT, or CE revealed findings considered confirmatory of a diagnosis of SBT, the results were classified in the "definite SBT" category. When findings were suggestive but not confirmatory of SBT, the results were classified as "suspicious SBT." When no evidence of neoplasia was found, the results were classified as "negative." Locations of SBTs were classified as duodenum, jejunum (proximal, mid, or distal), and ileum (proximal, mid, or distal). In patients operated on, the locations of SBTs were classified according to operative findings. In patients nonoperated on, we approximately estimated the locations of SBTs with the depth of insertion of enteroscope, the size of small intestinal lumen, and the shape of villi and folds.

2.1. Capsule Endoscopy. The PillCam SB video (SB1 and SB2, Given Imaging, Yokneam, Israel) and MiroCam (IntroMedic, Seoul, South Korea) were used for CE. Polyethylene glycol solution $(2-4 \mathrm{~L})$ was administered before the examination for cleansing and enhancement of visual clarity. Video findings were interpreted by experienced gastrointestinal endoscopists at each center.
TABLE 1: Clinical characteristics of patients with small bowel tumors and characteristics of small bowel tumors.

\begin{tabular}{lc}
\hline Clinical characteristics & $N(\%)$ \\
\hline M:F & $50: 29$ \\
Age (years, mean \pm SD) & $47.2 \pm 20.2$ \\
Mean duration of symptoms (days \pm SD) & $168.9 \pm 966.6$ \\
Smoking (current) & $13(16.4 \%)$ \\
Alcohol (current) & $17(21.5 \%)$ \\
Comorbidity & \\
$\quad$ Hypertension & $7(8.9 \%)$ \\
$\quad$ Diabetes mellitus & $2(2.5 \%)$ \\
Liver cirrhosis & $1(1.3 \%)$ \\
$\quad$ Others & $14(17.8 \%)$ \\
Hb (g/dL, mean \pm SD) & $10.1 \pm 2.9$ \\
Anemia & $45(57 \%)$ \\
Protein (g/dL, mean \pm SD) & $6.1 \pm 0.9$ \\
Albumin (g/dL, mean \pm SD) & $3.7 \pm 0.6$ \\
Characteristics of small bowel tumors & \\
Single tumor & $54(68.3 \%)$ \\
Size of tumor (cm, mean \pm SD) & $3.5 \pm 2.6$ \\
Extents & \\
$\quad$ Localized & $68(86.1 \%)$ \\
$\quad$ Locally advanced & $6(7.6 \%)$ \\
$\quad$ Metastasis & $5(6.3 \%)$ \\
\hline
\end{tabular}

2.2. Double Balloon Enteroscopy. DBE was performed using EN-450P5/20 or EN-450T5 (Fujinon Inc., Saitama, Japan) under conscious sedation. All procedures were carried out by experienced endoscopists. The technique for insertion has been previously described [19]. Enteroscopic exploration was discontinued when the targeted lesion was reached. Total enteroscopy was confirmed by reaching the cecum, or by reaching an India ink stain previously made through the other approach. Biopsy, polypectomy, or endoscopic mucosal resection specimens obtained by $\mathrm{DBE}$ were reviewed by experienced pathologists. The protocol was approved by the Institutional Review Board at each institute.

2.3. Statistical Analysis. SPSS 11.5 (SPSS Inc., Chicago, IL) was used for all statistics. Continuous variables are presented as means \pm standard deviations and were analyzed using ANOVA. The comparisons of clinical factors affecting diagnostic yields of SBTs by CT, SBFT, and CE were assessed by using chi-square test and Fisher exact test. The proportions of patients with positive findings at two examinations were compared, and a significant difference between the tests was calculated by using the exact McNemar test. A $P$ value $<0.05$ was considered statistically significant.

\section{Results}

3.1. Clinicopathologic Characteristics of SBTs. The clinical characteristics of patients are presented in Table 1 . The incidence of SBTs was higher in men $(63.3 \%)$ than in women 


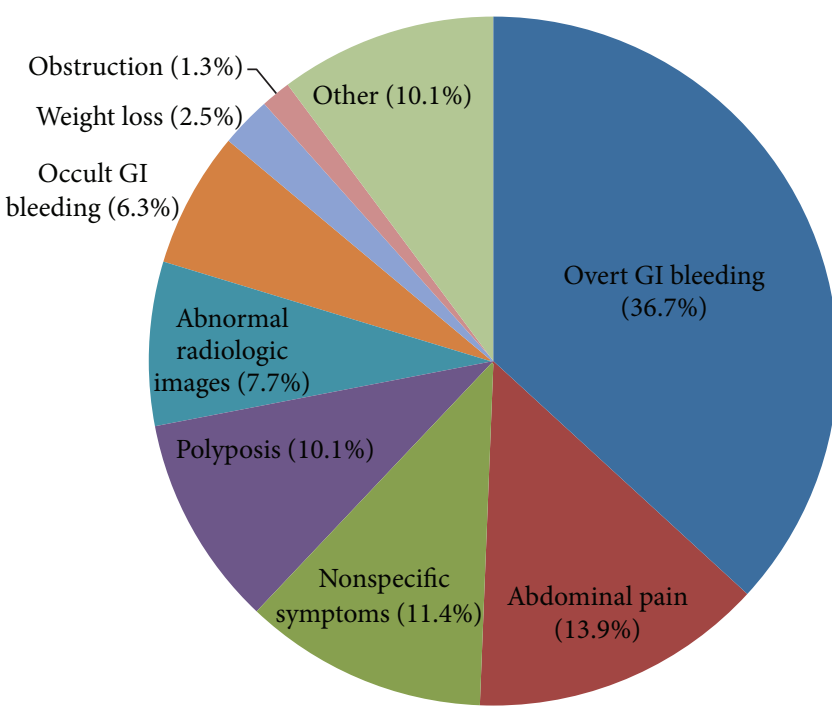

Figure 1: Various indications of diagnostic tests for small bowel tumors.

(36.7\%). Forty-five (57\%) of 79 patients had anemia, and the mean hemoglobin level was $10.1 \pm 2.9 \mathrm{~g} / \mathrm{dL}$. The mean size of SBTs was $3.5 \pm 2.6 \mathrm{~cm}$, and 54 patients $(68.3 \%)$ had a single lesion. SBTs were localized in 68 patients $(86.1 \%)$ and locally advanced in 6 patients (7.6\%) and metastatic disease was present in 5 patients $(6.3 \%)$. The most frequent symptom was overt GI bleeding $(36.7 \%)$ and abdominal pain (13.9\%) (Figure 1). Of 43 patients who were treated by surgery, 29 patients underwent DBE and surgery and 14 patients underwent surgery without DBE. The size of tumors in the patients with DBE and surgery was significantly smaller than the patients diagnosed by surgery without DBE $(3.31 \pm$ $2.33 \mathrm{~cm}$ versus $5.88 \pm 2.93 \mathrm{~cm}, P=0.031$ ).

3.2. Locations of SBTs according to Diagnosis. The SBTs were benign in 63 patients $(79.7 \%)$ and malignant in 16 patients (20.3\%). Benign tumors were leiomyoma (24/52, 46.2\%), hamartoma $(17 / 52,32.7 \%)$, benign polyps (7/52, 13.5\%), lipoma (3/52, 5.8\%), and hemangioma (1/51, 2.9\%). Malignant tumors were adenocarcinoma $(7 / 16,43.8 \%)$, gastrointestinal stromal tumor (GIST) $(5 / 16,31.3 \%)$, lymphoma $(3 / 16,18.8 \%)$, and carcinoid tumor $(1 / 16,6.3 \%)$. Both benign and malignant tumors occurred most frequently in the proximal jejunum $(34 / 79,43.0 \%)$ followed by the distal ileum $(9 / 79,11.4 \%)$, duodenum $(8 / 79,10.1 \%)$, middle jejunum $(6 / 79,7.6 \%)$, distal jejunum $(5 / 79,6.3 \%)$, proximal ileum $(4 / 79,5.1 \%)$, and middle ileum $(3 / 79,3.8 \%)$.

3.3. Diagnostic Yields of SBFT, CT, and CE for SBTs. The diagnostic yields for definitive SBTs were $55.8 \%$ (38/68) in CT, $46.1 \%$ (18/39) in SBFT, and 83.3\% (45/54) in CE (Table 2). Using DBE as a reference in the $95 \%$ confidence interval (CI), the sensitivity of each diagnostic method was $40.4 \%$ (95\% CI, 27.01-54.90\%) in CT, 43.9\% (95\% CI, 28.47-60.25\%) in SBFT, and 79.6\% (95\% CI, 65.66-89.76\%) in CE. A significant difference was found between diagnostic yields of CE and
TABLE 2: Comparison of diagnostic yields of CE and other radiologic studies.

\begin{tabular}{lcccc}
\hline & CT & SBFT & CE & DBE \\
\hline Definitive & $38(55.8 \%)$ & $18(46.1 \%)$ & $45(83.3 \%)$ & $61(93.8 \%)$ \\
Suspicious & $20(29.4 \%)$ & $9(23.1 \%)$ & $2(3.7 \%)$ & \\
Negative & $10(14.7 \%)$ & $12(30.7 \%)$ & $7(12.9 \%)$ & $4(6.2 \%)$ \\
Total & 68 & 39 & 54 & 65 \\
\hline
\end{tabular}

CE: capsule endoscopy; SBFT: small bowel follow-through; CT: computed tomography; DBE: double balloon enteroscopy.

TABLE 3: Comparison of diagnostic yields of capsule endoscopy and other radiologic studies.

\begin{tabular}{lccc}
\hline & Difference (\%) & $95 \%$ CI (\%) & $P$ value \\
\hline CE versus SBFT & 36.36 & $9.36-50.64$ & 0.0075 \\
CE versus CT & 33.33 & $12.75-43.82$ & 0.0015 \\
\hline
\end{tabular}

CE: capsule endoscopy; SBFT: small bowel follow-through; CT: computed tomography.

TABLE 4: Clinical factors affecting diagnostic yields of each modality.

\begin{tabular}{lcccc}
\hline \multicolumn{2}{c}{ Clinical factors } & CT & SBFT & CE \\
\hline \multirow{3}{*}{ Anemia } & Presence & $52.5 \%$ & $48 \%$ & $75 \%$ \\
& Absence & $16.2 \%$ & $30 \%$ & $85.7 \%$ \\
& $P$ value & 0.019 & 0.333 & 0.638 \\
\hline \multirow{3}{*}{ Size of tumor } & $<10 \mathrm{~mm}$ & $30 \%$ & $14.2 \%$ & $77.7 \%$ \\
& $>10 \mathrm{~mm}$ & $37.5 \%$ & $46.1 \%$ & $60 \%$ \\
& $P$ value & 0.900 & 0.043 & 0.624 \\
\hline \multirow{3}{*}{ Main symptoms } & Bleeding & $57.6 \%$ & $50 \%$ & $66.6 \%$ \\
& Nonbleeding & $42.4 \%$ & $50 \%$ & $68.9 \%$ \\
\hline Location & $P$ value & 0.021 & 0.458 & 0.615 \\
\hline
\end{tabular}

CE: capsule endoscopy; SBFT: small bowel follow-through; CT: computed tomography.

SBFT (36.36\%; 95\% CI, 9.36-50.64\%; $P=0.0075)$ and between those of CE and CT (33.33\%, 95\% CI, 12.75-43.82\%; $P=0.0015$ ) (Table 3 ). The clinical factors affecting diagnostic yields were the presence of anemia $(P=0.019)$ and bleeding $(P=0.043)$ for CT and tumor size $>10 \mathrm{~mm}(P=0.021)$ for SBFT (Table 4). There were no statistically significant clinical factors affecting diagnostic yields of CE.

There were 32 patients who had all three modalities (CT, SBFT, and CE). We compared the sensitivities of these three modalities in 32 patients. It showed that the sensitivity of $\mathrm{CE}$ for SBTs is significantly higher than SBFT or CT (Table 5).

3.4. Characteristics of SBTs Missed by CE. In nine (16.7\%) of 54 patients, SBTs were not detected by CE but were eventually diagnosed by DBE. Seven patients had negative findings and two patients had suspicious findings on CE. SBTs $>10 \mathrm{~mm}$ were identified in six patients $(66.7 \%)$. Most of the missed SBTs were located in the proximal jejunum $(5,55.6 \%)$, followed by the proximal ileum $(2,22.2 \%)$, middle jejunum (1, 11.1\%), and distal jejunum $(1,11.1 \%)$. The final diagnoses in these nine patients were GIST $(4,44.4 \%)$, small polyps (3, 
TABLE 5: Comparisons of sensitivities of three modalities for small bowel tumors.

\begin{tabular}{|c|c|c|c|c|c|}
\hline & & $\mathrm{CE}$ & & Totol & $D_{\text {pul }}$ \\
\hline & Definitive & Suspicious & Negative & lotal & $P$ value \\
\hline CT & & & & & 0.005 \\
\hline Definitive & 10 & 1 & 1 & 12 & \\
\hline Suspicious & 13 & 0 & 5 & 18 & \\
\hline Negative & 1 & 1 & 0 & 2 & \\
\hline Total & 24 & 2 & 6 & 32 & \\
\hline SBFT & & & & & 0.046 \\
\hline Definitive & 10 & 0 & 3 & 13 & \\
\hline Suspicious & 5 & 1 & 1 & 7 & \\
\hline Negative & 9 & 1 & 2 & 12 & \\
\hline Total & 24 & 2 & 6 & 32 & \\
\hline
\end{tabular}

CE: capsule endoscopy; SBFT: small bowel follow-through; CT: computed tomography.

$33.3 \%)$, adenocarcinoma (1, 11.1\%), and inflammatory fibroid polyp $(1,11.1 \%)$.

\section{Discussion}

In this study, we demonstrated that the most common SBT was leiomyoma for benign tumor and adenocarcinoma for malignancy, and the most common location of SBTs was proximal jejunum in Korea. The diagnostic yield and sensitivity of CE was higher than SBFT and CT scan. We also found that the SBTs missed by CE were $16.7 \%$, missed SBTs were mostly located in proximal jejunum, and GIST was the most common histologic type of missed SBTs.

Primary small bowel neoplasms are rare accounting for only $3 \%$ of all gastrointestinal tract neoplasia in the United States [20]. In Korea, the 2008 annual report of cancer statistics showed that small intestinal cancer accounted for $0.98 \%$ of all GI malignancies [21]. However, several studies have reported an increasing incidence of SBTs [22, 23]. SBTs are often diagnosed too late for successful treatment because clinical manifestations of SBTs are usually nonspecific and conventional radiologic tests have some limitations to detect small sized SBTs.

The study from the United States showed that carcinoid tumor is the most common SBTs rather than adenocarcinoma [24]. Cangemi et al. reported that the most common SBT was carcinoid tumor (19.4\%) followed by GIST and lymphoma [25]. They also reported that hamartoma (10.4\%) was the most common benign tumor, followed by inflammatory polyps and adenoma. In Japan, lymphoma (21.5\%) and GIST (18.8\%) were the most common tumors, and carcinoid tumors were rare $(2.8 \%)$ [26]. In recent Korean studies, carcinoid tumor is rare in the small intestine $[27,28]$. This difference is presumed to be a result of genetic and ethnic factors. Primary adenocarcinoma is the most common SBT detected by DBE in another Japanese study [29]. In a Korean study of 112 patients with SBTs, the most common malignancy was GIST/leiomyoma and the most common benign polyp was hamartoma [29]. In our study, leiomyoma and GIST were categorized separately, and the most common benign SBT was leiomyoma. Adenocarcinoma was the most common malignancy in our study. Further studies are needed to accurately determine the frequency of SBTs in Korea.

The most common location of SBTs is the ileum in a previous study of the United States [25]. Our study found that the distribution of SBTs is similar to that of a previous Korean study, which reported that SBTs were most frequently located in the jejunum (61.7\%), followed by ileum $(34.9 \%)$ and duodenum (16.1\%) [29].

Diagnostic radiology studies such as SBFT, enteroclysis, and CT were traditionally used to diagnose SBTs. SBFT is relatively easy to perform and is tolerable for patients. However, the sensitivity of SBFT is $30-44 \%$. Enteroclysis has a better diagnostic yield, but it requires a highly skilled radiologist and may cause significant discomfort for patients. Despite the advances in CT technology, conventional CT scans still detect only large tumors greater than $10 \mathrm{~mm}$ in diameter. In comparison, the diagnostic yield of $\mathrm{CE}$ was superior to those of CT and SBFT $[30,31]$. The diagnostic impact of CE for SBTs was reported as 52.6\% [22]. CE also allows endoscopic imaging of the entire small bowel without discomfort or exposure to radiation. However, CE is contraindicated for patients with suspected or documented intestinal obstruction and presents no opportunity for histological confirmation or therapeutic intervention. Our results demonstrated that $\mathrm{CE}$ has a higher diagnostic yield and sensitivity than those of other radiologic modalities.

We demonstrated that the miss rate of CE for SBTs was $16.5 \%$. We also found that missed tumors were most commonly located in the proximal jejunum (55.6\%). A metaanalysis including 24 studies and over 500 patients found that the overall miss rate of $\mathrm{CE}$ for small bowel mass lesions was $18.6 \%$ [32]. Ross et al. reported that CE identified the mass lesion in only 5 (33\%) of 15 patients with SBTs [17]. There are several possible explanations for nondetection of SBTs by CE. SBTs are usually single, whereas most patients with angiodysplasia or ulcers have multiple lesions. SBTs are usually located in the proximal small bowel and may be missed because of tumbling or excessive capsule transfer velocity due to excessive bowel peristalsis. Therefore, the detection of SBTs by CE remains a challenge despite CE being a major advance in small bowel evaluation.

Our study is limited by its retrospective design. Furthermore, because our study included only patients with proven SBTs, the specificity of each diagnostic method could not be analyzed. There are limitations to measure accurate sensitivity or miss rate of CE because the patients with negative CE results were more likely not to undergo DBE or surgery.

Taken together, we concluded that CE shows higher diagnostic yield and sensitivity for the diagnosis of SBTs compared with other radiologic tests. However, CE can miss some significant tumors, particularly those located in the proximal jejunum. Further endoscopic investigation by deep enteroscopy should be considered for patients with nonspecific findings on $\mathrm{CE}$ but high clinical suspicion for SBT. 


\section{Conflict of Interests}

The authors have no commercial associations that might represent a conflict of interests in relation to this paper.

\section{Authors' Contribution}

Jung Wan Han and Sung Noh Hong equally contributed as the first author.

\section{Acknowledgments}

This work was supported by the National Research Foundation of Korea (NRF) grant funded by the Korean government (NRF-2014R1A2A1A11052136). The authors would like to thank Small Intestinal Research Group of the Korean Association for the Study of Intestinal Diseases (KASID) for participation and assistance.

\section{References}

[1] B. G. O'Riordan, M. Vilor, and L. Herrera, "Small bowel tumors: an overview," Digestive Diseases, vol. 14, no. 4, pp. 245-257, 1996.

[2] A. J. Minardi Jr., G. B. Zibari, D. F. Aultman, R. W. McMillan, and J. C. McDonald, "Small-bowel tumors," Journal of the American College of Surgeons, vol. 186, no. 6, pp. 664-668, 1998.

[3] R. F. Thoeni and R. G. Gould, "Enteroclysis and small bowel series: comparison of radiation dose and examination time," Radiology, vol. 178, no. 3, pp. 659-662, 1991.

[4] S. S. Gill, D. M. Heuman, and A. A. Mihas, "Small intestinal neoplasms," Journal of Clinical Gastroenterology, vol. 33, no. 4, pp. 267-282, 2001.

[5] A. Maieron, D. Hubner, B. Blaha et al., "Multicenter retrospective evaluation of capsule endoscopy in clinical routine," Endoscopy, vol. 36, no. 10, pp. 864-868, 2004.

[6] J. S. Barkin and C. O’Loughlin, "Capsule endoscopy contraindications: complications and how to avoid their occurrence," Gastrointestinal Endoscopy Clinics of North America, vol. 14, no. 1, pp. 61-65, 2004.

[7] R. de Franchis, E. Rondonotti, C. Abbiati, G. Beccari, and C. Signorelli, "Small-bowel malignancy," Gastrointestinal Endoscopy Clinics of North America, vol. 14, no. 1, pp. 139-148, 2004.

[8] G. M. Cobrin, R. H. Pittman, and B. S. Lewis, "Increased diagnostic yield of small bowel tumors with capsule endoscopy," Cancer, vol. 107, no. 1, pp. 22-27, 2006.

[9] A. A. Bailey, H. S. Debinski, M. N. Appleyard et al., "Diagnosis and outcome of small bowel tumors found by capsule endoscopy: a three-center Australian experience," American Journal of Gastroenterology, vol. 101, no. 10, pp. 2237-2243, 2006.

[10] E. Estévez, B. González-Conde, J. L. Vázquez-Iglesias, P. A. Alonso, M. D. L. A. Vázquez-Millán, and R. Pardeiro, "Incidence of tumoral pathology according to study using capsule endoscopy for patients with obscure gastrointestinal bleeding," Surgical Endoscopy and Other Interventional Techniques, vol. 21, no. 10, pp. 1776-1780, 2007.

[11] D. Urbain, D. de Looze, I. Demedts et al., "Video capsule endoscopy in small-bowel malignancy: a multicenter Belgian study," Endoscopy, vol. 38, no. 4, pp. 408-411, 2006.

[12] A. Trifan, A.-M. Singeap, C. Cojocariu, C. Sfarti, and C. Stanciu, "Small bowel tumors in patients undergoing capsule endoscopy: a single center experience," Journal of Gastrointestinal and Liver Diseases, vol. 19, no. 1, pp. 21-25, 2010.

[13] R. Urgesi, M. E. Riccioni, A. Bizzotto et al., "Increased diagnostic yield of small bowel tumors with PillCam: the role of capsule endoscopy in the diagnosis and treatment of gastrointestinal stromal tumors (GISTs). Italian single-center experience," Tumori, vol. 98, no. 3, pp. 357-363, 2012.

[14] A. M. Sîngeap, A. Trifan, C. Cojocariu, C. Sfarti, and C. Stanciu, "Capsule endoscopy role in diagnosis of small bowel tumors," Revista Medico-Chirurgicala a Societatii de Medici si Naturalisti din Iasi, vol. 114, no. 3, pp. 988-992, 2010.

[15] A. Koulaouzidis, E. Rondonotti, A. Giannakou, and J. N. Plevris, "Diagnostic yield of small-bowel capsule endoscopy in patients with iron-deficiency anemia: a systematic review," Gastrointestinal Endoscopy, vol. 76, no. 5, pp. 983-992, 2012.

[16] A. Madisch, W. Schimming, F. Kinzel et al., "Locally advanced small-bowel adenocarcinoma missed primarily by capsule endoscopy but diagnosed by push enteroscopy," Endoscopy, vol. 35, no. 10, pp. 861-864, 2003.

[17] A. Ross, S. Mehdizadeh, J. Tokar et al., "Double balloon enteroscopy detects small bowel mass lesions missed by capsule endoscopy," Digestive Diseases and Sciences, vol. 53, no. 8, pp. 2140-2143, 2008.

[18] E. S. Zagorowicz, A. M. Pietrzak, E. Wronska et al., "Small bowel tumors detected and missed during capsule endoscopy: single center experience," World Journal of Gastroenterology, vol. 19, no. 47, pp. 9043-9048, 2013.

[19] H. Yamamoto, Y. Sekine, Y. Sato et al., "Total enteroscopy with a nonsurgical steerable double-balloon method," Gastrointestinal Endoscopy, vol. 53, no. 2, pp. 216-220, 2001.

[20] R. Siegel, D. Naishadham, and A. Jemal, "Cancer statistics, 2013," CA: Cancer Journal for Clinicians, vol. 63, no. 1, pp. 11-30, 2013.

[21] Korea Central Cancer Registry and National Cancer Center, Annual Report of Cancer Statistics in Korea in 2008, Ministry of Health and Welfare of Korea, Seoul, Republic of Korea, 2010.

[22] D. Y. Cheung, I.-S. Lee, D. K. Chang et al., "Capsule endoscopy in small bowel tumors: a multicenter Korean study," Journal of Gastroenterology and Hepatology, vol. 25, no. 6, pp. 1079-1086, 2010.

[23] E. Rondonotti, M. Pennazio, E. Toth et al., "Small-bowel neoplasms in patients undergoing video capsule endoscopy: a multicenter European study," Endoscopy, vol. 40, no. 6, pp. 488495, 2008.

[24] K. Y. Bilimoria, D. J. Bentrem, J. D. Wayne, C. Y. Ko, C. L. Bennett, and M. S. Talamonti, "Small bowel cancer in the United States: changes in epidemiology, treatment, and survival over the last 20 years," Annals of Surgery, vol. 249, no. 1, pp. 63-71, 2009.

[25] D. J. Cangemi, M. K. Patel, V. Gomez, J. R. Cangemi, M. E. Stark, and F. J. Lukens, "Small bowel tumors discovered during double-balloon enteroscopy: analysis of a large prospectively collected single-center database," Journal of Clinical Gastroenterology, vol. 47, no. 9, pp. 769-772, 2013.

[26] K. Mitsui, S. Tanaka, H. Yamamoto et al., "Role of doubleballoon endoscopy in the diagnosis of small-bowel tumors: the first Japanese multicenter study," Gastrointestinal Endoscopy, vol. 70, no. 3, pp. 498-504, 2009.

[27] Y. B. Park, J. I. Kim, B. H. Ha et al., "Endoscopic treatment of gastrointestinal carcinoid tumors," The Korean Journal of Medicine, vol. 73, no. 3, pp. 274-282, 2007. 
[28] J. H. Chang, S. W. Kim, W. C. Chung et al., "Clinical review of gastrointestinal carcinoid tumor and analysis of the factors predicting metastasis," The Korean Journal of Gastroenterology, vol. 50, no. 1, pp. 19-25, 2007.

[29] H. Imaoka, N. Higaki, T. Kumagi et al., "Characteristics of small bowel tumors detected by double balloon endoscopy," Digestive Diseases and Sciences, vol. 56, no. 8, pp. 2366-2371, 2011.

[30] B.-I. Lee, H. Choi, K.-Y. Choi et al., "Clinical characteristics of small bowel tumors diagnosed by double-balloon endoscopy: KASID multi-center study," Digestive Diseases and Sciences, vol. 56, no. 10, pp. 2920-2927, 2011.

[31] W. Honda, N. Ohmiya, Y. Hirooka et al., "Enteroscopic and radiologic diagnoses, treatment, and prognoses of small-bowel tumors," Gastrointestinal Endoscopy, vol. 76, no. 2, pp. 344-354, 2012.

[32] G. Costamagna, S. K. Shah, M. E. Riccioni et al., "A prospective trial comparing small bowel radiographs and video capsule endoscopy for suspected small bowel disease," Gastroenterology, vol. 123, no. 4, pp. 999-1005, 2002. 


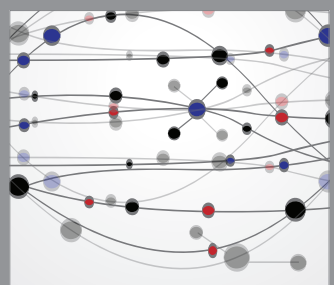

The Scientific World Journal
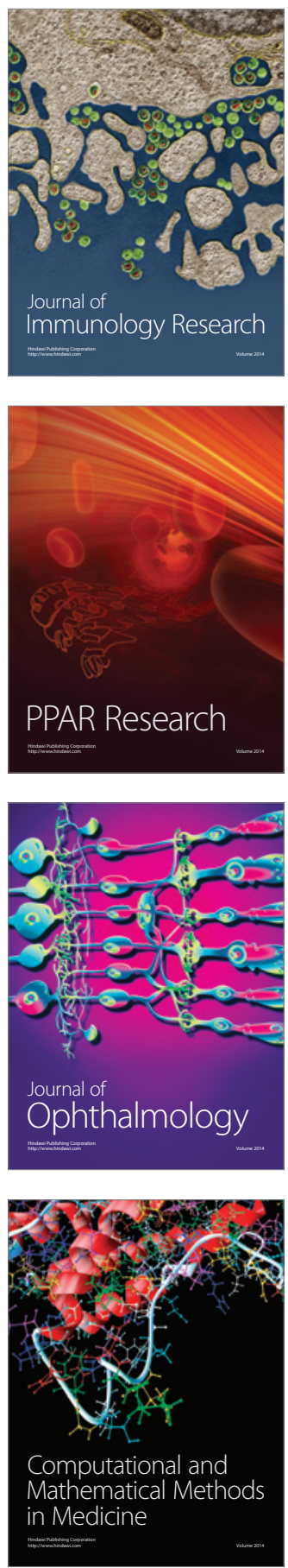

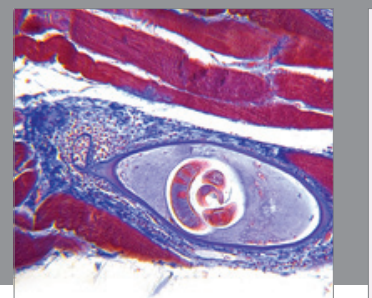

Gastroenterology

Research and Practice
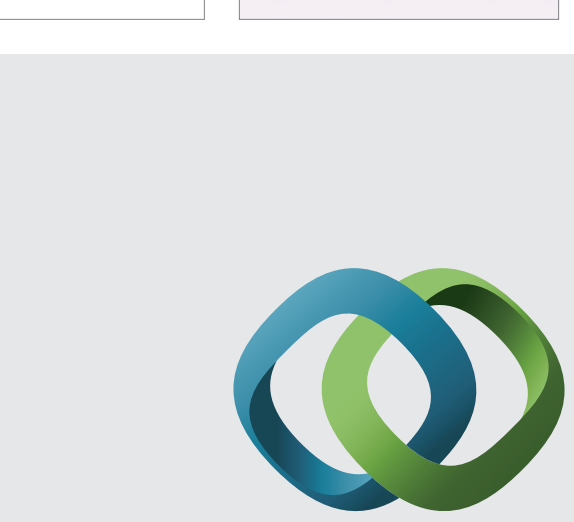

\section{Hindawi}

Submit your manuscripts at

http://www.hindawi.com
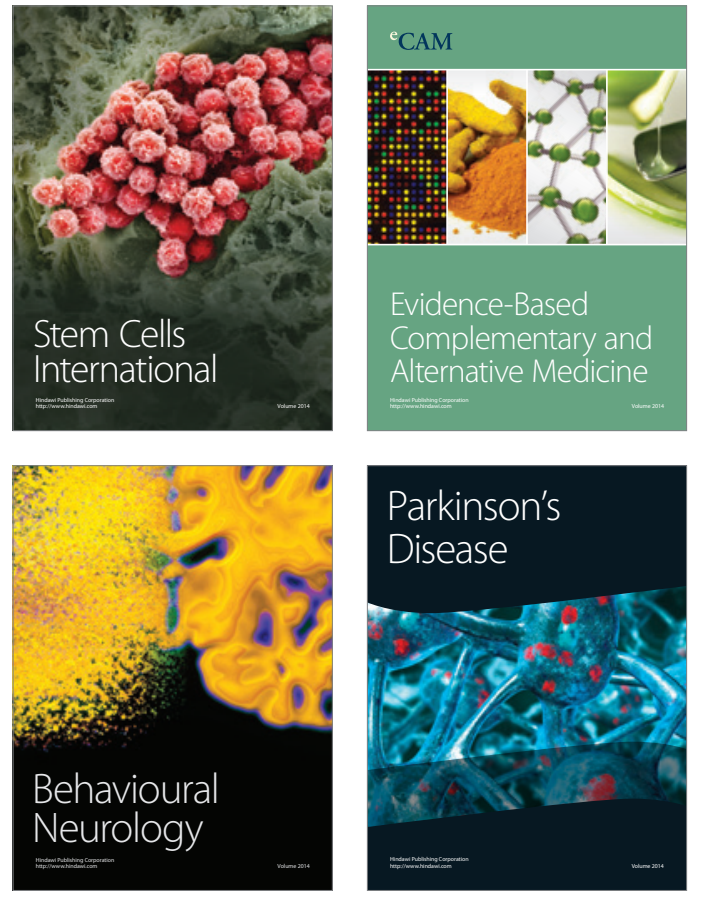
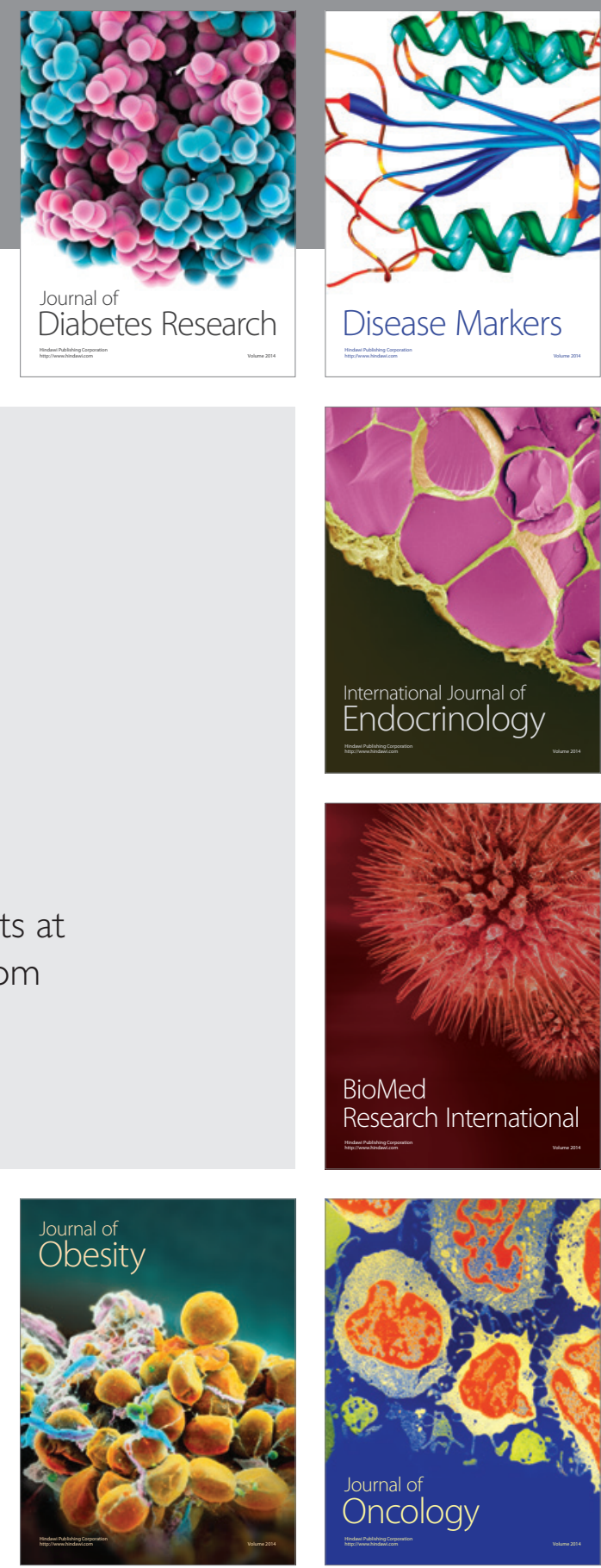

Disease Markers
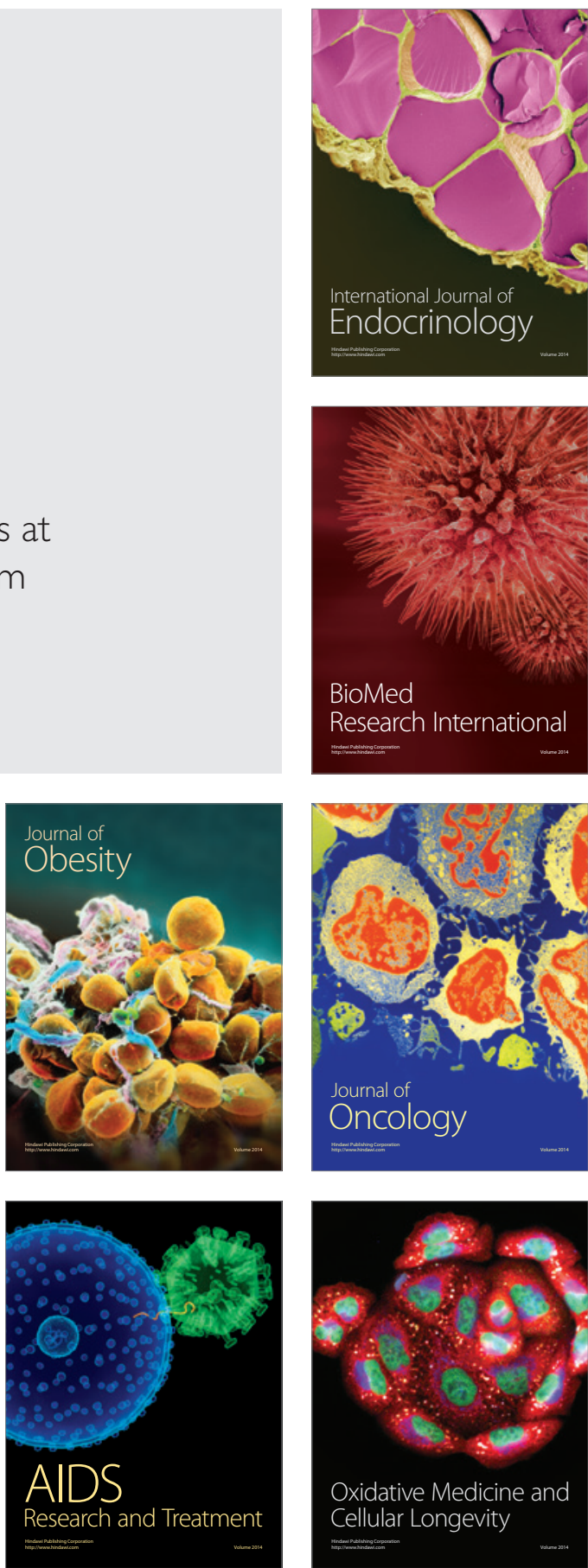